\title{
AN EXPERIMENTAL TEST OF A CORE REBT HYPOTHESIS: EVIDENCE THAT IRRATIONAL BELIEFS LEAD TO PHYSIOLOGICAL AS WELL AS PSYCHOLOGICAL AROUSAL
}

\author{
Steven Harris \\ The Crouch Hall Road Surgery, London, UK
}

\author{
Martin F. Davies \\ Windy Dryden \\ Goldsmiths College, University of London, UK \\ Address correspondence to Dr. Steven Harris, The Crouch Hall Road Surgery, Crouch End, N8 8HJ London, \\ UK; e-mail: steveharrisoo1@yahoo.com.
}

\begin{abstract}
While numerous studies support Rational Emotive Behavior Therapy and Theory (REBT), they tend to be limited by their use of correlational designs, simulated scenarios and self-report measures. This study tested a core REBT hypothesis in an experimental design using multiple physiological as well as psychological measures. Ninety patients from a medical practice were placed in a real-life stressful situation while holding either a rational, an irrational, or an indifference belief. Those holding a rational belief reported the greatest increase in concern whereas those holding an irrational belief reported the greatest increase in anxiety. Of particular significance, those holding a rational belief showed a decrease in systolic blood pressure whereas those holding an irrational belief showed an increase (diastolic blood pressure increased in both conditions). These results not only support the core REBT hypothesis, but also suggest a way to differentiate between beliefs and emotions by measuring physiological as well as psychological changes.
\end{abstract}

\section{KEY WORDS}

REBT; physiological arousal; emotions; beliefs. 
A central hypothesis of Rational Emotive Behavior Therapy and Theory (REBT) is that irrational beliefs lead to psychological disturbance. Numerous studies have examined this hypothesis, typically finding a significant correlation between measures of irrational beliefs and measures of emotional distress such as anxiety, depression and low self-esteem (e.g., Daly \& Burton, 1983; Goldfried \& Sobocinski, 1975; Nelson, 1977). For example, Goldfried and Sobocinsky (1975) found that people holding irrational beliefs about imagined social rejection scenes reported greater anxiety and hostility than did people who did not hold these beliefs.

While such studies provide support for the core REBT hypothesis, they tend to employ a limited range of methodologies. The studies are correlational rather than true experimental designs. The participants tend to be college students. The procedures tend to be artificial and unreal: Participants are often asked to imagine a stressful event rather than to face an actual one; yet, according to Ellis (1994), irrational beliefs may not be triggered in relatively 'safe' imaginary situations. The measures commonly used to assess irrational beliefs in these studies often have poor discriminant validity. For example, items on the Irrational Belief Test (Jones, 1968) and the Rational Behavior Inventory (Shorkey \& Whiteman, 1977) measure emotions as well as beliefs. One consequence of this is that the results of studies using such measures may merely reflect a confounding of beliefs and emotions in the item content of scales rather than a real association between cognition and affect (Zurawski \& Smith, 1987). A small number of studies have attempted to address some of these limitations by employing measures of emotional and physiological arousal. Craighead, Kimball, and Rekah (1979) attempted to replicate the Goldfried and Sobocinski study with measures of physiological arousal and self-statements in addition to self-report measures of mood change. Physiological measures included respiration, skin conductance, heart rate and finger pulse volume. The results showed that 'high-irrational' subjects emitted more negative self-statements, but there were no significant changes on physiological measures. Master and Gershman (1983) also looked at the relationship between irrational beliefs and physiological arousal. They found that at high levels of problem relevance there was a significantly greater galvanic skin response (GSR) in direct response to the stimulus situation and also to irrational statements rather than to rational and control statements, but they explained these differences in terms of conditioning rather than REBT theory.

While the above studies may represent some advance in methodology, they failed to clearly define emotional and physiological arousal. For example, blood pressure was not measured despite being a key indicator of physiological arousal and little consideration was given to the definition and meaning of the various emotional terms. Dryden (2002) describes an important distinction between emotional terms such that healthy and unhealthy negative emotions are distinguished by their cognitive consequences and action tendencies. According to this distinction, anxiety is an unhealthy negative emotion which leads to an unrealistic appraisal of and physical withdrawal from a situation, whereas concern-its healthy counterpart-leads to a realistic appraisal and more constructive action.

In terms of physiological measures, blood pressure (systolic and diastolic) is the most important and most common variable studied in emotion and arousal. It is well established that acute stress and anxiety can lead to a transient increase in blood pressure via changes in cardiac output and vascular resistance regulated by the autonomic nervous system (e.g., Shapiro, Goldstein, \& Jamner, 1996; Whitehead, Blackwell, De Silva, \& Robinson, 1994); chronic stress and anxiety can induce chronic hypertension (e.g., Jonsson \& Hanson, 1977; Noyes, Clancy, Hoenk, \& Slymen, 1980). A large number of studies involving Cognitive Behavior Therapy (CBT) have found significant increases in blood pressure (systolic and/or diastolic) but no significant changes in heart rate or respiratory rate in response to stress or anxiety (e.g., Asmundson \& Stein, 1994; Zeller, Handschin, Gyr, Maritna, \& Battegay, 2004). Most of these studies have focused on the effect of cognitive-behavioral stress-reduction techniques on blood pressure in patients with hypertension and/or a history of previous mental illness such as anxiety disorders (e.g., Eisenberg Delbanco, \& Berkey, 1993). Very few studies have investigated changes in a stressful situation 
using normotensive patients or patients with no previous history of mental illness and no studies have employed REBT techniques.

The present experiment investigated the core hypothesis of REBT-that irrational beliefs lead to psychological disturbance-while addressing most of the limitations of previous studies, but in addition investigated physiological indicators of disturbance. The main hypothesis was that, in a real-life stressful situation, holding an irrational belief would lead to increased blood pressure (systolic and/or diastolic), while holding a rational belief would lead either to no change or even to a decrease in blood pressure (systolic and/or diastolic). At the same time, holding an irrational belief would lead to an increase in anxiety while holding a rational belief would lead to an increase in concern compared with holding an indifference belief (control condition).

\section{METHOD}

\section{Participants}

Participants were randomly selected over a 2-week period from a North London General Practice patient population who were visiting their GP for a regular appointment. All patients were screened for a history of cardiovascular disease or mental disorders; a total of nine patients were excluded on these criteria. A further nine were excluded because they gave a low rating (under $70 \%$ ) in the belief they were asked to hold. Only one patient refused to participate in the study. A total of 90 patients (56 women, 34 men) aged 18-64 comprised the final sample.

\section{Measures and Procedure}

At the start of the procedure, patients first had their blood pressure, heart rate and respiratory rate measured as part of a routine health check. Blood pressure (systolic and diastolic) and heart rate were measured using a fully automated BP device (Omron M7 BP monitor). Respiratory rate was measured by the GP over a period of thirty seconds. They were then asked if they would participate in a brief psychological study examining the interaction between psychology and physiology. Those who agreed to participate signed a consent form. Patients were then seated in a comfortable chair facing a video camera placed on a stand at eye level and asked to keep as still as possible for a period of 1 minute. They were told that the video would be scrutinized by a panel of 10 'behavioral experts' who would examine their faces for signs of movement (including expression, eye movement and nasal flaring) and that they would receive a performance score out of a hundred for stillness.

Patients were randomly allocated to hold one of three beliefs during the video recording. In the rational belief condition $(\mathrm{n}=30)$, patients were asked to hold the belief: "I would prefer to perform well in this presentation, but I don't have to. If I don't, then I would not like it, but I would not be less worthy for my performance. Rather, I am a fallible complex human being, too complex to be rated on the basis of my performance." In the irrational belief condition ( $\mathrm{n}=$ 30), patients were asked to hold the belief: "I must perform well in this presentation. If I don't, then I'm a failure!" In the indifference belief condition ( $n=30)$, patients were asked to hold the belief: "I do not care how I perform in this presentation."

After the video recording procedure, patients rated changes in their levels of anxiety and concern (defined in terms of the cognitive consequences and action tendencies described by Dryden (2002)) on 9-point self-report scales and had their blood pressure, heart rate and respiration rate measured again. Finally, all patients rated their conviction in the belief they had been asked to hold on a percentage scale. 


\section{RESULTS}

There were no significant differences between the three belief conditions with respect to age or gender $(p>.05, n s)$. There were significant effects on systolic and diastolic blood pressure of age, $F(1,83)=16.43, p<.001$ and $F(1,83)=9.91, p<.005$ respectively. Gender had a marginally significant effect on systolic blood pressure only, $F(1,83)=3.14, p=.08$.

Type of belief had a significant main effect on increase in anxiety, $F(2,83)=12.14, p<.001$, and increase in concern, $F(2,83)=7.37, p<.001$. As can be seen from Figure 1, patients holding an irrational belief showed the greatest increase in anxiety $(M=+2.23)$ while patients holding a rational belief showed the greatest increase in concern $(M=+1.83)$.

Systolic and diastolic blood pressure increased from before to after the video recording, $F(1,83)=$ $1.57, p<.15$, and $F(1,83)=6.59, p<.01$, respectively. However, these main effects were qualified by significant interactions with type of belief, $F(2,83)=3.90, p<.025$, and $F(2,83)=2.60, p=$ .08. As can be seen from Figure 2, for systolic blood pressure, patients holding an irrational belief showed a significant increase from before to after the video recording $(M=+5.40)$, whereas patients holding a rational belief showed a significant decrease $(M=-4.40)$. As can be seen from Figure 3, for diastolic blood pressure, patients holding an irrational belief showed a much larger increase from before to after the video recording $(M=+6.73)$ than patients holding a rational belief $(M=+2.57)$. Patients holding an indifference belief showed no significant changes in systolic or diastolic blood pressure $(p>.05, \mathrm{~ns})$.

Increase in systolic blood pressure was found to correlate with increase in diastolic blood pressure $(r=.48, p<.001)$ and both correlated significantly with increase in anxiety $(r=.43, p<.001$ and $r=.61, p<.001$ ) while only increase in diastolic blood pressure was found to correlate (negatively) with increase in concern $(r=-.21, p<.05)$.

Analysis of heart rate showed no significant differences as a function of type of belief. Similarly, analysis of respiratory rate showed no significant differences.

\section{DISCUSSION}

The results of this experiment confirmed the main hypothesis. Patients holding irrational beliefs showed an increase in systolic blood pressure, while those holding rational beliefs showed a decrease in systolic blood pressure (both groups showed an increase in diastolic blood pressure). At the same time, patients holding an irrational belief reported an increase in anxiety while those holding a rational belief reported an increase in concern (patients holding an indifference belief showed no significant changes).

These results therefore support the core hypothesis of REBT that beliefs are at the core of psychological disturbance. Furthermore, they suggest an objective way to differentiate between the variables of cognition and affect. Thus, irrational thinking may be described as holding a rigid belief with an elevation in systolic blood pressure, whereas rational thinking may be described as holding a flexible belief with a reduction in systolic blood pressure. Anxiety may be defined in terms of emotional arousal along with elevation in systolic blood pressure, whereas concern may be defined in terms of cognitive arousal along with reduction in systolic blood pressure.

While Master and Gershman (1983) found a significantly greater GSR (a measure of physiological arousal) in direct response to irrational statements than to rational and control statements, they explained the differences in terms of classical conditioning rather than REBT. The present 
experiment suggests an explanation in terms of REBT. Irrational statements, derived from rigid and absolutistic thinking, lead to a rise in systolic blood pressure defined by an increase in peripheral vascular resistance (autonomic rigidity). Rational statements, derived from flexible and relativistic thinking, lead to a drop in systolic blood pressure defined by a decrease in peripheral vascular resistance (autonomic flexibility). In other words, mental rigidity leads to autonomic rigidity and mental flexibility leads to autonomic flexibility.

Furthermore, this experiment supports a qualitative rather than quantitative distinction (Ellis \& DiGiuseppe, 1993) between anxiety and concern in line with the distinction between healthy and unhealthy negative emotions (e.g. Dryden, 2002). It suggests that anxiety and concern do not lie along a continuum, but can be differentiated by type of arousal (emotional versus cognitive) along with change in systolic blood pressure (an increase versus a decrease). According to Bond and Dryden (1996) the core hypotheses of REBT are untestable because Ellis described cognitions, emotions and behavior as interdependent and therefore cannot be analyzed separately. The findings of this experiment, that cognitions and emotions can be analyzed separately, may therefore have implications for the testability of REBT theory.

There are several important limitations to this study. The participants were recruited from a North London General Practice serving an affluent population which may not be considered representative of the general population. Participants were asked to participate in the study immediately after a regular consultation with their GP so that measurements may have been contaminated by the content of the consultation itself. Thus, experimenter expectancies may have had an effect as well as the GP-patient relationship which may have introduced a respondent bias (e.g. Heppner, Kivlighan, \& Wampold, 1999).

There are important implications of the present findings for health interventions. High blood pressure is the commonest medical diagnosis in the community with up to $20 \%$ of the population affected at any one time. It is a well-known risk factor for a wide range of medical conditions including heart attack and stroke. In terms of psychological interventions, most of the literature has focused on stressreducing techniques which employ CBT to help reduce blood pressure (e.g., Vocks, Ockenfels, Jurgensen, Mussgay, \& Ruddel, 2004). These techniques have been shown to have some effect on reduction of blood pressure in hypertensive and/or anxious patients. Very few studies have examined CBT techniques for the prevention of onset of hypertension in normotensive populations and none has employed REBT techniques. The results of this study suggest that REBT may have a key role to play in the management and prevention of hypertension.

In conclusion, the present study supports the core hypothesis of REBT and suggests how future studies might better test REBT empirically. It is hoped that this study and others like it will help stimulate more experimental research on REBT using a wider range of measures so that it may be seen more clearly to be a valid therapeutic technique both by the scientific community and the community at large.

\section{REFERENCES}

Asmundson, G. J., \& Stein, M. B. (1994). Vagal attenuation in panic disorder: an assessment of parasympathetic nervous system function and subjective reactivity to respiratory manipulations. Psychosomatic Medicine, 56, 187-193.

Bond, F. W., \& Dryden, W. (1996). Why two, central REBT hypotheses appear untestable. Journal of Rational Emotive and Cognitive Behaviour Therapy, 14, 29-40. 
Craighead, W. E., Kimball, W., \& Rehak, P. (1979). Mood changes, physiological responses, and self-statements during social rejection imagery. Journal of Consulting and Clinical Psychology, $47,385-396$.

Daly, J. J., \& Burton, R. L. (1983). Self esteem and irrational beliefs: an exploratory investigation with implications for counselling. Journal of Counselling Psychology, 30, 361-366.

Dryden, W. (2002). Rational emotive behaviour therapy; a reader. London: SAGE.

Eisenberg, D. M., Delbanco, T. L., \& Berkey, C. S. (1993). Cognitive behavioural techniques for hypertension. Annals of Internal Medicine, 118, 964-972.

Ellis A. (1994). Reason and emotion in psychotherapy (rev. edn.). Secaucus, NJ: Birch Lane.

Ellis, A., \& DiGiuseppe, R. (1993). Are inappropriate or dysfunctional feelings in Rational-Emotive Therapy qualitative or quantitative? Cognitive Therapy and Research, 17(5), 471-477.

Goldfried, M., \& Sobocinski, D. (1975). Effect of irrational beliefs on emotional arousal. Journal of Consulting and Clinical Psychology, 43, 504-510.

Heppner, P., Kivlighan, D., \& Wampold, B. (1999). Research design in counselling (2nd edn.). Belmont: Wadsworth Publishing Company.

Jones, R. A. (1968). A factored measure of Ellis' irrational belief system with personality and maladjustment correlates. Dissertation Abstracts International, 29, 4379-4380.

Jonsson, A., \& Hanson, L. (1977). Prolonged exposure to a stressful stimulus (noise) as a cause of raised blood pressure in man. Lancet, 1, 86-87.

Master, S., \& Gershman, L. (1983). Physiological responses to rationalemotive self-verbalizations. Journal of Behavior Therapy and Experimental Psychiatry, 14, 289-296.

Nelson, R. (1977). Irrational beliefs and depression. Journal of Consulting and Clinical Psychology, 45, 1190-1191.

Noyes, R., Clancy, J., Hoenk, T. R., \& Slymen, D. J. (1980). The prognosis of anxiety neurosis. Archives General Psychiatry, 37, 173-176.

Shapiro, D., Goldstein, I. B., \& Jamner, L. D. (1996). Effects of cynical hostility, anger out, and defensiveness on ambulatory blood pressure in black and white college students. Psychosomatic Medicine, 58, 354-364.

Shorkey, C. T., \& Whiteman, V. L. (1977). Development of the rational behavior inventory: initial validity and reliability. Educational and Psychological Measurement, 37, 527-534.

Vocks, S., Ockenfels, M., Jurgensen, R., Mussgay, L., \& Ruddel, H. (2004). Blood pressure reactivity can be reduced by a cognitive behavioural stress management program. International Journal of Behavioral Medicine, 11, 63-70.

Whitehead, W. E., Blackwell, B., De Silva, H., \& Robinson, A. (1994). Anxiety and anger in hypertension. Journal of Psychosomatic Research, 21, 383-389.

Zeller, A., Handschin, D., Gyr, M. B., \& Battegay, E. (2004). Blood pressure and heart rate of students undergoing a medical licensing examination. Blood Pressure, 13, 20-24.

Zurawski, R. M., \& Smith, T. W. (1987). Assessing irrational beliefs and emotional distress: 
evidence and implications of limited discriminant validity. Journal of Consulting Psychology, 34, 224-227. 
Figure 1. Increases in anxiety and concern as a function of belief type.
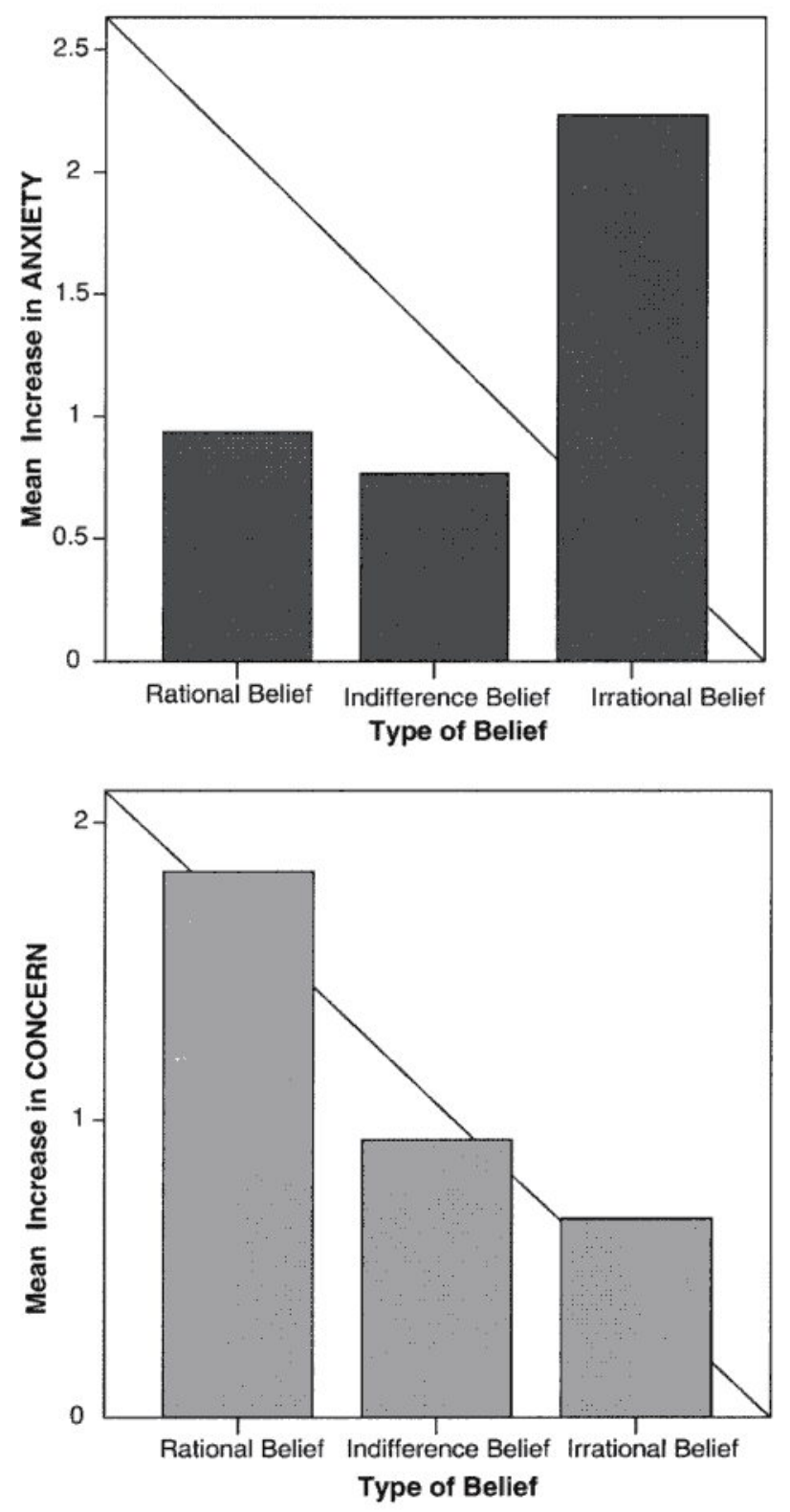
Figure 2. Systolic Blood Pressure before/after the video recording as a function of belief type.
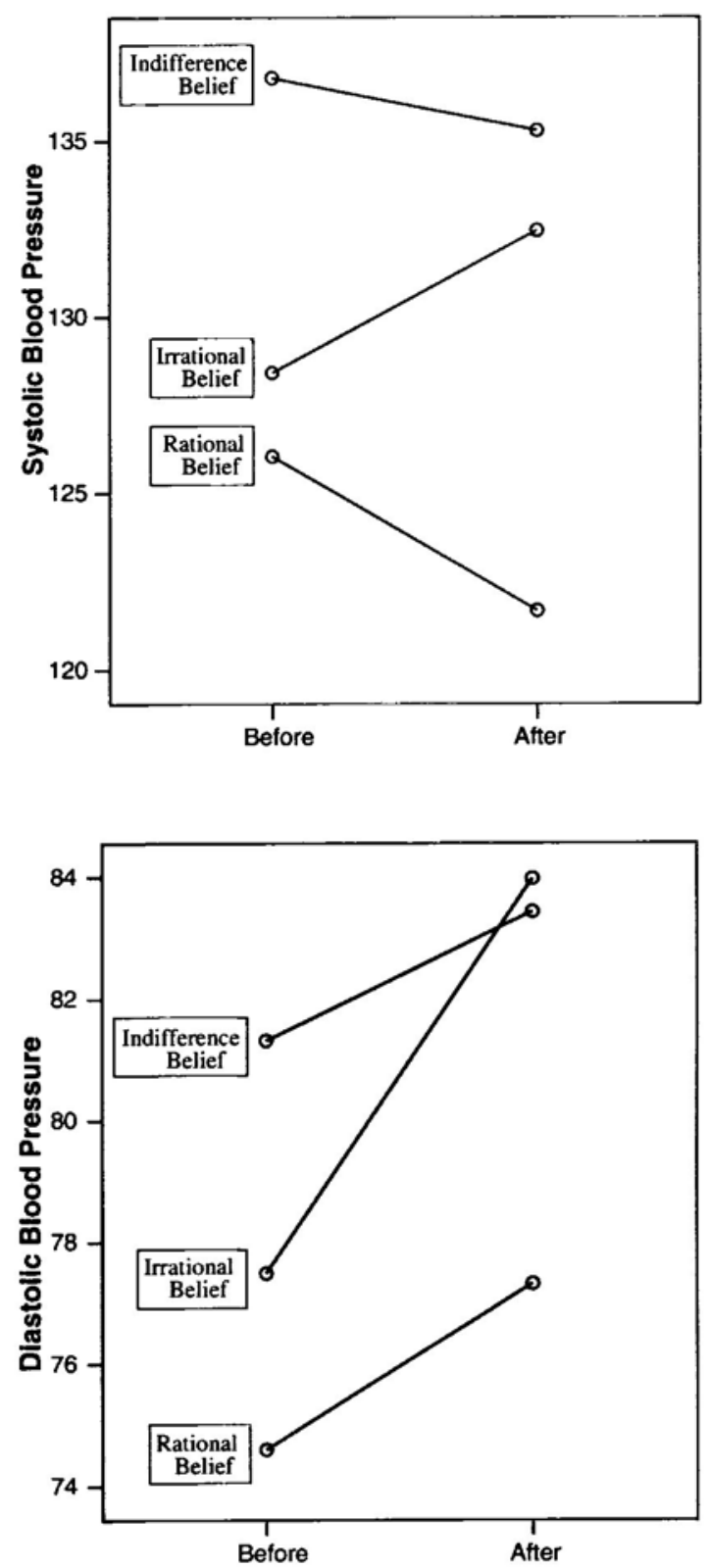

Figure 3. Diastolic Blood Pressure before/after the video recording as a function of belief type. 